\title{
Lipocalin-Like Prostaglandin D Synthase but Not Hemopoietic Prostaglandin D Synthase Deletion Causes Hypertension and Accelerates Thrombogenesis in Mice ${ }^{\mathbf{S}}$
}

\author{
Wen-Liang Song, ${ }^{1,2}$ Emanuela Ricciotti, ${ }^{1}$ Xue Liang, ${ }^{3}$ Tilo Grosser, Gregory R. Grant, \\ and Garret A. FitzGerald
}

Department of Systems Pharmacology and Translational Therapeutics (W.-L.S., E.R., X.L., T.G., G.A.F.), Institute for Translational Medicine and Therapeutics (W.-L.S., E.R., X.L., T.G., G.R.G., G.A.F.), and Perelman School of Medicine and Department of Genetics (G.R.G.), University of Pennsylvania, Philadelphia, Pennsylvania

Received May 25, 2018; accepted September 17, 2018

\section{ABSTRACT}

Prostaglandin (PG) $D_{2}$ is formed by two distinct PGD synthases (PGDS): lipocalin-type PGDS (L-PGDS), which acts as a $\mathrm{PGD}_{2}$ producing enzyme and as extracellular lipophilic transporter, and hematopoietic PGDS (H-PGDS), a $\sigma$ glutathione-S-transferase. $\mathrm{PGD}_{2}$ plays an important role in the maintenance of vascular function; however, the relative contribution of L-PGDS- and $\mathrm{H}$-PGDS-dependent formation of $\mathrm{PGD}_{2}$ in this setting is unknown. To gain insight into the function played by these distinct PGDS, we assessed systemic blood pressure (BP) and thrombogenesis in L-Pgds and H-Pgds knockout (KO) mice. Deletion of L-Pgds depresses urinary $\mathrm{PGD}_{2}$ metabolite (PGDM) by $\sim 35 \%$, whereas deletion of $\mathrm{H}$-Pgds does so by $\sim 90 \%$. Deletion of $\mathrm{L}-\mathrm{Pgds}$, but not $\mathrm{H}$-Pgds, elevates $\mathrm{BP}$ and accelerates the thrombogenic occlusive response to a photochemical injury to the carotid artery. HQL-79, a H-PGDS inhibitor, further depresses PGDM in L-Pgds KO mice, but has no effect on BP or on the thrombogenic response. Gene expression profiling reveals that pathways relevant to vascular function are dysregulated in the aorta of L-Pgds KOs. These results indicate that the functional impact of L-Pgds deletion on vascular homeostasis may result from an autocrine effect of L-PGDS-dependent $\mathrm{PGD}_{2}$ on the vasculature and/or the L-PGDS function as lipophilic carrier protein.

\section{Introduction}

Prostaglandin (PG) $\mathrm{D}_{2}$ is synthesized in both the central nervous system and the peripheral tissues (Ricciotti and FitzGerald, 2011). In the brain, $\mathrm{PGD}_{2}$ is involved in the regulation of sleep and other neurologic activities, including pain perception (Ricciotti and FitzGerald, 2011). In peripheral tissues, $\mathrm{PGD}_{2}$ is produced mainly by mast cells and other cells, including platelets, macrophages, and lymphocytes, and there it plays a role in inflammatory and atopic diseases, although it might also exert an array of immunologically mediated antiinflammatory functions (Ricciotti and FitzGerald, 2011).

$\mathrm{PGD}_{2}$ is formed by the action of either lipocalin-like PGD synthase (L-PGDS) or hemopoietic PGD synthase (H-PGDS) (Urade and Hayaishi, 2000). L-PGDS, known also as $\beta$ trace protein, is a member of the lipocalin superfamily, a group of

This work was supported by National Institutes of Health [Grant HL117798 to G.A.F.]. G.A.F. is the McNeil Professor of Translational Medicine and Therapeutics.

${ }^{1}$ W.-L.S. and E.R. should be considered joint first authors.

${ }^{2}$ Current affiliation: Vanderbilt University Medicine Center, Cardiology Division of Department Medicine, Nashville, Tennessee.

${ }^{3}$ Current affiliation: Merck Research Laboratories Cambridge Exploratory Science Center, Cambridge, Massachusetts.

https://doi.org/10.1124/jpet.118.250936.

S This article has supplemental material available at jpet.aspetjournals.org secretory proteins that bind and transport a variety of lipophilic molecules (Herlong and Scott, 2006). H-PGDS is a $\sigma$ class glutathione-S-transferase family member (Urade and Eguchi, 2002). L-PGDS is expressed mainly in the central nervous system, retina, male and female genital organs, heart, and vasculature, whereas H-PGDS is generally localized to the cytosol of immune and inflammatory cells. $\mathrm{PGD}_{2}$ exerts it biologic effects via two $G$ protein-coupled receptors: the prostaglandin D receptor (DP) and the chemoattractant receptor-homologous molecule expressed on Th2 cells (CRTH2) (Sawyer et al., 2002; Hirai et al., 2003; Kabashima and Narumiya, 2003).

There is increasing evidence that PGD synthase (PGDS) and $\mathrm{PGD}_{2}$ play a relevant role in the modulation of the vascular function (Ricciotti and FitzGerald, 2011). $\mathrm{PGD}_{2}$ mediates vasodilation and increases vascular permeability (Braun and Schror, 1992), relaxation of vascular and nonvascular smooth muscle (Narumiya and Toda, 1985; Hall, 2000), reduction of ocular pressure (Goh et al., 1988), inhibition of platelet aggregation (Song et al., 2012), and chemotactic recruitment of inflammatory cells (Herlong and Scott, 2006).

L-PGDS expression is induced by laminar shear stress via activator protein 1 in vascular endothelial cells (Taba et al., 2000; Miyagi et al., 2005), and it is expressed in synthetic smooth muscle cells in the intimal of atherosclerotic lesions

ABBREVIATIONS: BP, blood pressure; DEG, differentially expressed gene; GO, gene ontology; H-PGDS, hemopoietic PGDS; KO, knockout; L-PGDS, lipocalin-like PGDS; LPS, lipopolysaccharide; PG, prostaglandin; PGDM, PGD 2 metabolite; PGDS, PGD synthase; WT, wild-type. 
and in coronary plaques of arteries with severe stenosis (Eguchi et al., 1997; Hirawa et al., 2002). Patients with stable angina present significantly higher plasma level of L-PGDS in the cardiac vein than in the coronary artery, and its concentration in the cardiac vein decreases immediately after angioplasty (Eguchi et al., 1997). Human serum L-PGDS level increases with the progression of atherosclerosis, and it is an independent predictor of coronary severity (Inoue et al., 2008). Moreover, elevated serum L-PGDS levels are associated with the presence of atrial fibrillation in hypertensive patients (Yalcin et al., 2016).

More recently, it has been reported that serum level of L-PGDS may represent a biomarker of kidney function (White et al., 2015), pregnancy-induced hypertension (Duan et al., 2016), and active lupus nephritis (Brunner et al., 2017).

In mice, L-PGDS deletion accelerates atherogenesis and induced glucose intolerance and obesity (Ragolia et al., 2005; Tanaka et al., 2009). $\mathrm{PGD}_{2}$ also plays a relevant role in the evolution of vascular inflammation. In the inflamed intima, $\mathrm{PGD}_{2}$ is partly produced by H-PGDS-producing inflammatory cells that are chemotactically attracted to the vasculature (Herlong and Scott, 2006). $\mathrm{PGD}_{2}$ has been shown to inhibit expression of proinflammatory genes, such as inducible nitric oxide synthase and plasminogen activator inhibitor1 in vascular cells (Nagoshi et al., 1998; Negoro et al., 2002).

The major objective of the present study was to elucidate the relative contributions of L-PGDS- and H-PGDS-derived $\mathrm{PGD}_{2}$ to blood pressure (BP) homeostasis and thrombogenesis in mice. Our results indicate that H-PGDS makes a more substantial contribution than L-PGDS to $\mathrm{PGD}_{2}$ biosynthesis, as reflected by the major urinary $\mathrm{PGD}_{2}$ metabolite. Deletion of L-Pgds, but not of H-Pgds, predisposed both to higher BP and increased thrombogenesis.

\section{Materials and Methods}

Animals. All animal protocols were approved by the University of Pennsylvania Institutional Animal Care and Use Committee. L-Pgds knockout mice [L-Pgds knockout (KO) provided by Y. Urade, Osaka, Japan] and H-Pgds knockout mice (H-Pgds KO provided by Y. Kanaoka, Boston, MA) were all on a C57BL/6 background. Hence, wild-type (WT) littermate controls were used in both sets of studies.

HQL-79, a H-PGDS inhibitor, was purchased from Tocris Bioscience (Minneapolis, MN).

WT and L-Pgds KO mice received HQL-79 $(30 \mathrm{mg} / \mathrm{kg}$ per day in $0.5 \%$ methyl cellulose) by oral gavage for 10 days, as previously reported (Aritake et al., 2006; Mohri et al., 2009).

Peritoneal Macrophage Culture. Peritoneal macrophages were collected 4 days after i.p. injection of $0.5 \mathrm{ml} 10 \%$ thioglycolate (SigmaAldrich, St. Louis, MO). Nonadherent cells were removed after 2 hours of incubation. Adherent cells were treated with $5 \mu \mathrm{g} / \mathrm{ml}$ lipopolysaccharide (LPS; Sigma-Aldrich, St. Louis, MO) for 4 and 24 hours.

Mass Spectrometric Analysis of Prostanoids and Their Metabolites. Prostanoids or their metabolites were measured by mass spectrometry, as described previously (Song et al., 2007). Briefly, macrophage production of $\mathrm{PGD}_{2}$ was determined by quantification of $\mathrm{PGD}_{2}$ in cell culture supernatants and normalized with total protein content. Systemic production of $\mathrm{PGE}_{2}, \mathrm{PGD}_{2}$, thromboxane (Tx) $\mathrm{A}_{2}$, and prostacyclin was determined by quantification of their major urinary metabolites (7-hydroxy-5,11-diketotetranorprostane-1,16dioic acid [PGEM], 11,15-dioxo- $9 \alpha$-hydroxy-, 2,3,4,5-tetranorprostan1,20-dioic acid [tetranor PGDM], 2,3-dinor $\mathrm{TxB}_{2}$ [TXM], and 2,3-dinor 6-keto $\mathrm{PGF}_{1 \alpha}$ [PGIM], respectively) in 24-hour collections and normalized with creatinine.

BP Measurement by Tail Cuff. Resting systolic BP was measured in conscious WT, L-Pgds KOs, and H-Pgds KOs, 8- to 12-weekold mice, using a computerized noninvasive tail cuff system (Visitech Systems, Apex, NC). Mice were trained to adapt to the system for 14 days. After that, BP was recorded daily for 3 consecutive days at the same time of the day and in the same way. Data were collected and analyzed using updated BP-2000 software (Visitech Systems).

Photochemical Injury of the Carotid Artery. L-Pgds KO, H-Pgds KO mice, and their littermate WT control mice underwent photochemically induced vascular injury in the carotid artery, as previously reported (Cheng et al., 2006).

Briefly, in anesthetized (sodium pentobarbital, $80 \mathrm{mg} / \mathrm{kg}$ ) mice 12-16 weeks of age, the left common carotid artery was isolated, and a Doppler flow probe (model 0.5 VB; Transonic Systems, Ithaca, NY) was applied. The probe was connected to a flowmeter (model T105; Transonic Systems) and interpreted with a computerized data acquisition program (PowerLab; AD Instruments, Colorado Springs, CO). Rose Bengal (Fisher Scientific International, Norwalk, CT) was injected into the jugular vein in a volume of $0.12 \mathrm{ml}$ in a final concentration of $50 \mathrm{mg} / \mathrm{kg}$. Vascular injury was induced by applying $1.5 \mathrm{~mW}$ green light laser (540 nm; Melles Griot, Carlsbad, CA) at a distance of $5 \mathrm{~cm}$ from the desired site on the carotid artery. Blood flow was monitored for 120 minutes or until stable occlusion occurred. Stable occlusion was defined as a blood flow of $0 \mathrm{ml} / \mathrm{min}$ for 3 minutes. Mice that did not occlude within the 120-minute time course were excluded from the experiment. Complete and $50 \%$ occlusion time were determined.

Microarray Analysis. Thoracic aortas were harvested from male WT and L-Pgds KO mice, 8-12 weeks old. Total RNA was prepared using TRIzol reagent (Invitrogen, Carlsbad, CA) and RNeasy columns (Qiagen, Valencia, CA). Samples were prepared in one batch using the Nugen sample preparation protocol and hybridized to Affymetrix MOE430 v2 (Santa Clara, CA). Data from the CEL files were summarized and normalized using print-tip loess algorithm from the Bioconductor array package for R.6 Patterns of Gene Expression software, which uses the false discovery rate method and was used to identify statistically significant differentially expressed gene (DEG)s, as previously described (Grant et al., 2005). The false discovery rate was set at $10 \%$ for all differential gene expression analyses, so that $90 \%$ of the predicted genes are expected to be true positives. The expression data were clustered (hierarchical method) and visualized using a modified version of the heatmap\{stats\} function in $R$.

Ingenuity Pathways Analysis (www.ingenuity.com; Ingenuity Systems, Redwood City, CA) was employed to identify relevant canonical pathways enriched by DEGs between WT and L-Pgds KO mice.

Gene ontology (GO) enrichment analysis was employed to identify enriched GO biologic functions (http://geneontology.org/page/ go-enrichment-analysis).

Microarray data are available in the Gene Expression Omnibus database (http://www.ncbi.nlm.nih.gov/geo/) under accession number GSE77642.

Real-Time Polymerase Chain Reaction Analysis. Total RNAs isolated from aortas, brain, adrenal gland, and heart (as described above) were reverse-transcribed into cDNA by Taqman reverse-transcription reagents (Applied Biosystems, Foster City, CA). TaqMan gene expression assays (Applied Biosystems, Foster City, CA) for phenylethanolamine N-methyltransferase (Pnmt, Mm00476993_m1), L-PGDS (Mm01330513_m1), H-PGDS (Mm00479848), DP1 (Mm00436050_m1), and DP2 (Mm01223054_m1) were performed on an ABI Prism 7900 Sequence Detection System (Applied Biosystems, Foster City, CA). Results were normalized with 18s rRNA (Hs99999901_s1).

Statistics. Unless otherwise indicated, between-group comparison was performed using the nonparametric Mann-Whitney test or nonparametric one-way analysis of variance. All the comparisons were made between animals of the same age and gender on the same genetic background. A significance threshold of 0.05 was used, and it 
A

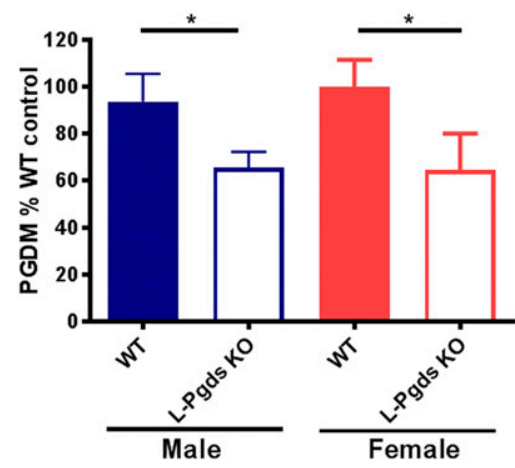

B

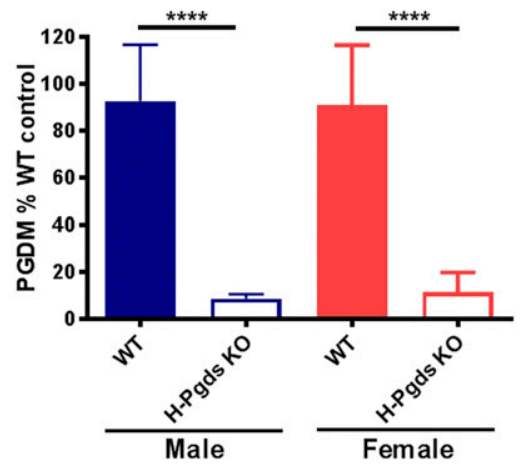

Fig. 1. Impact of L-Pgds and H-Pgds deletion on urinary $\mathrm{PGD}_{2}$ metabolite. (A) Urinary PGDM measured in WT and L-Pgds KO male (blue bars) and female (red bars) mice. (B) PGDM measured in WT and H-Pgds KO male (blue bars) and female (red bars) mice. Data in (A and B) are mean \pm S.E.M., $n=11-15 ; * P<0.05 ; * * * P<0.0001$ was indicated by a single asterisk. Significance of $<0.01$ is indicated by double asterisks on the graphs, and significance of $<0.001$ is indicated by triple asterisks. All data are presented as mean \pm S.E.M., unless otherwise stated.

\section{Results}

Differential Impact of L-Pgds and H-Pgds Deletion on Urinary PGD $_{2}$ Metabolite. Deletion of L-Pgds depressed $\mathrm{PGD}_{2}$ metabolite (PGDM) by $\sim 30 \%$ in both genders when compared with WT littermate controls (by $34.5 \%$ in males, $P<0.05, n=14-15$; by $35.3 \%$ in females, $P<0.05, n=$ 11; Fig. 1A). Deletion of H-Pgds had a more pronounced effect, depressing urinary PGDM by $\sim 90 \%$ (by $91.3 \%$ in males, $P<$ $0.0001 n=12$; by $87.8 \%$ in females, $P<0.0001, n=11-14$; Fig. 1B). There was no evidence of systemic rediversion of the $\mathrm{PGH}_{2}$ substrate, as urinary PGEM, TXM, and PGIM were unaltered in both mutants (Supplemental Fig. 1).

Deletion of H-Pgds, but Not L-Pgds, Suppresses PGD $_{2}$ Biosynthesis by Macrophages. H-Pgds was the primary source of $\mathrm{PGD}_{2}$ formation under basal conditions and after LPS-stimulated peritoneal macrophages (Supplemental Fig. 2). $\mathrm{PGD}_{2}$ production increased after 24 hours of LPS stimulation (Supplemental Fig. 2, A and B). Deletion of H-Pgds almost completely suppressed $\mathrm{PGD}_{2}$ production by macrophages (by $80 \%$ at baseline, $P<0.0001, n=9-15$; by
$98 \%$ at 24 hours of LPS stimulation, $P<0.0001, n=10-22$; Supplemental Fig. 2A). In contrast, deletion of L-Pgds had no impact on $\mathrm{PGD}_{2}$ production by macrophages (Supplemental Fig. 2B).

Deletion of L-Pgds, but Not H-Pgds, Elevates Blood Pressure. Systolic BP was measured by tail cuff in both male and female L-Pgds and H-Pgds KOs and their littermate controls. L-Pgds deletion caused a statistically significant increase in systolic BP in both females and males $(117.5 \pm 2.7$ vs. $126.3 \pm 2.5 \mathrm{mmHg}$ in females, $P<0.05, n=9-11 ; 119.3 \pm$ 2.4 vs. $131.2 \pm 4.2 \mathrm{mmHg}$ in males, $P<0.05, n=11-18$; Fig. 2 , $\mathrm{A}$ and $\mathrm{B})$. H-Pgds deletion did not cause any change in $\mathrm{BP}$ in either gender $(116.9 \pm 2.8$ vs. $112.9 \pm 2.8 \mathrm{mmHg}$ in females, $n=10-11 ; 111.7 \pm 5.4$ vs. $113.2 \pm 7.3 \mathrm{mmHg}$ in males, $n=$ 5-8; Fig. 2, C and D).

Deletion of L-Pgds, but Not H-Pgds, Accelerates the Thrombogenic Occlusive Response to a Photochemical Injury of the Carotid Artery. The time to complete common carotid artery occlusion after photochemical injury was reduced by L-Pgds deletion by approximately $45 \%$ in females and $30 \%$ in males $(68.6 \pm 7.1$ vs. $38.2 \pm 7.8$ minutes in females, $P<0.01, n=10-11 ; 59.0 \pm 4.0$ vs. $41.7 \pm 2.7$ minutes in males, $P<0.001, n=11-16$; Fig. 3, A and B). The time to complete common carotid artery occlusion in either gender was unaltered by H-Pgds deletion (Fig. 3, C and D).
A

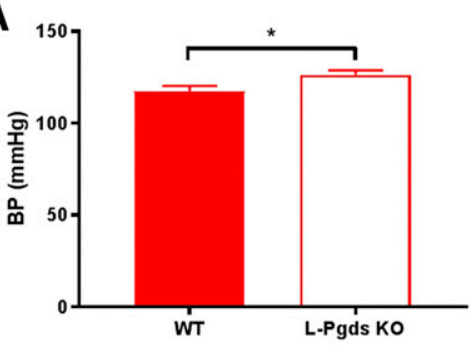

C

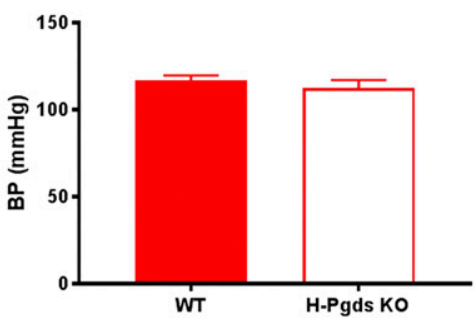

B

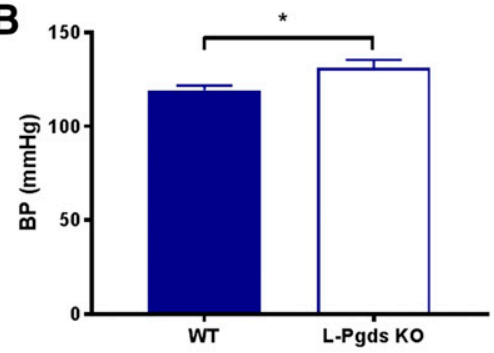

D

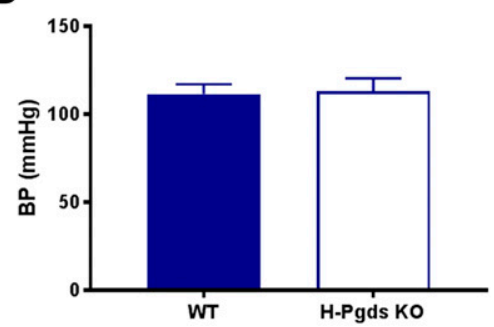

Fig. 2. Effect of L-Pgds deletion on systolic BP. (A) Systolic BP measured in WT and L-Pgds KO female mice. (B) BP measured in WT and L-Pgds KO male mice. (C) BP measured in WT and H-Pgds KO female mice. (D) BP measured in WT and H-Pgds KO male mice. Data are mean \pm S.E.M., $n=5-18 ;{ }^{*} P<0.05$. 

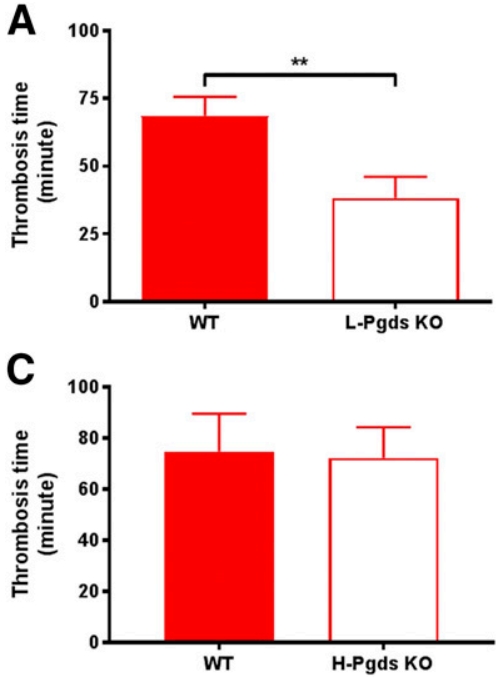

B

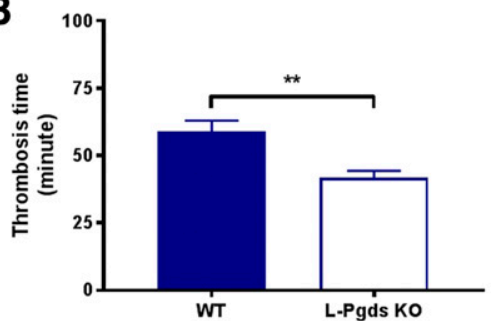

D

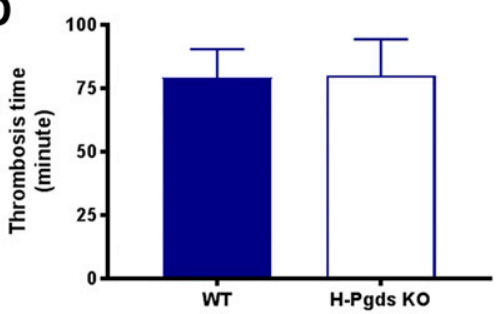

Fig. 3. Effect of L-Pgds deletion on photochemical-induced thrombogenesis in carotid artery. (A) Time to complete common carotid artery occlusion after photochemical injury (thrombosis time) measured in WT and L-Pgds KO female mice. (B) Thrombosis time measured in WT and L-Pgds KO male mice. (C) Thrombosis time measured in WT and H-Pgds KO female mice. (D) Thrombosis time measured in WT and H-Pgds KO male mice. Data are mean \pm S.E.M., $n=10-16 ; * * P<0.01$.
HQL-79, a Selective H-PGDS Inhibitor, Has No Effect on Blood Pressure or Thrombogenic Response. HQL79, a selective inhibitor of H-PGDS (Aritake et al., 2006; Mohri et al., 2009), caused a further reduction of PGDM level by approximately $30 \%$ in L-Pgds KOs $(8.9 \pm 0.9$ vs. $6.0 \pm$ $0.7 \mathrm{ng} / \mathrm{mg}$ creatinine, WT vs. L-Pgds KO + vehicle, $P<0.05$, $n=15-17 ; 6.0 \pm 0.7$ vs. $4.0 \pm 0.6 \mathrm{ng} / \mathrm{mg}$ creatinine, L-Pgds KO + vehicle vs. L-Pgds KO + HQL-79, $P<0.05, n=17$; Fig. 4A). The additional reduction of PGDM caused by HQL-79 administration in L-Pgds $\mathrm{KO}$ mice did not have any effect on BP (123.6 \pm 2.4 vs. $123.0 \pm 2.5 \mathrm{mmHg}$, L-Pgds $\mathrm{KO}+$ vehicle vs. L-Pgds KO + HQL-79, $n=18$; Fig. 4B) and on the thrombogenic response $(71.5 \pm 10.8$ vs. $63.0 \pm 6.2$ minutes, L-Pgds KO + vehicle vs. L-Pgds KO + HQL-79, $n=6-9$; Fig. 4C).

L-Pgds Deletion Causes Dysregulation of Genes Associated with Hypertension and Thrombosis. To gain insight into potential mechanisms by which L-Pgds deletion contributes to the hypertensive and prothrombotic phenotype, the transcriptome of aorta samples from L-Pgds KO was compared with those from WT mice with microarrays.
Deletion of L-Pgds has no effects on expression of H-Pgds, $\mathrm{PGD}_{2}$ receptors Ptgdr (DP1), and Ptgdr2 (DP2) (Supplemental Figs. 3 and 4). The analysis revealed a differential gene expression profile in the aorta from L-Pgds KO compared with control mice. In particular, a total of 454 probes (corresponding to 391 unique genes) was differentially expressed in the aorta of L-Pgds KO mice ( $q<0.1$; Supplemental Table 1), of which 186 were upregulated and 268 downregulated as compared with WTs (Fig. 5). As expected, the samples clustered according to their genotype, and the most downregulated gene in the aorta of L-Pgds KO mice was Ptgds (fold change of 40), which encodes the L-Pgds enzyme.

Among the genes upregulated in L-Pgds KO mice, we identified Pnmt (fold change of 1.5), a N-methyltransferase that methylates norepinephrine to form epinephrine. We confirmed an increased expression of Pnmt in the aorta, brain, and adrenal gland, but not in the heart of L-Pgds KO mice by reversetranscription polymerase chain reaction (Fig. 6). Pnmt's mRNA level was reported to be positively correlated with systemic BP (Reja et al., 2002), and indeed both male and
A
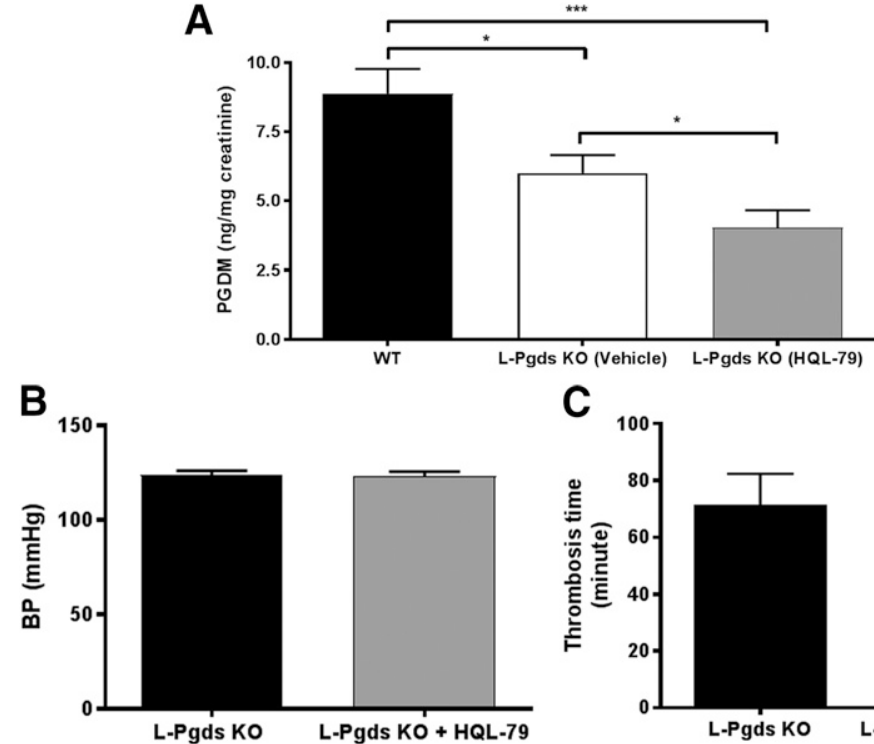

C

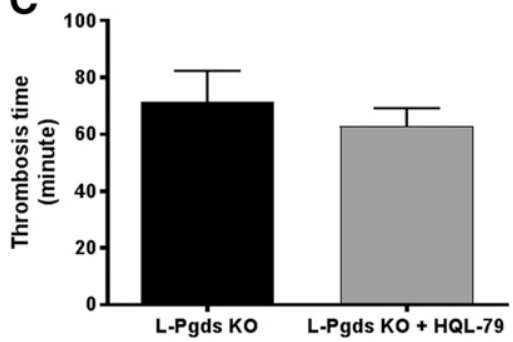

Fig. 4. Effect of HQL-79, a selective H-PGDS inhibitor, on urinary $\mathrm{PGD}_{2}$ metabolite, systolic $\mathrm{BP}$, and thrombogenesis in carotid artery in L-Pods KO mice. (A) Urinary PGDM measured in WT and in L-Pgds KO male mice treated with vehicle or HQL-79. (B) Systolic BP measured in L-Pgds KO male mice treated with vehicle or HQL-79. (C) Time to complete common carotid artery occlusion after photochemical injury (thrombosis time) measured in L-Pgds KO male mice treated with vehicle or HQL-79. Data are mean \pm S.E.M., $n=6-18 ; * P<0.05 ; * * * P<0.001$. 

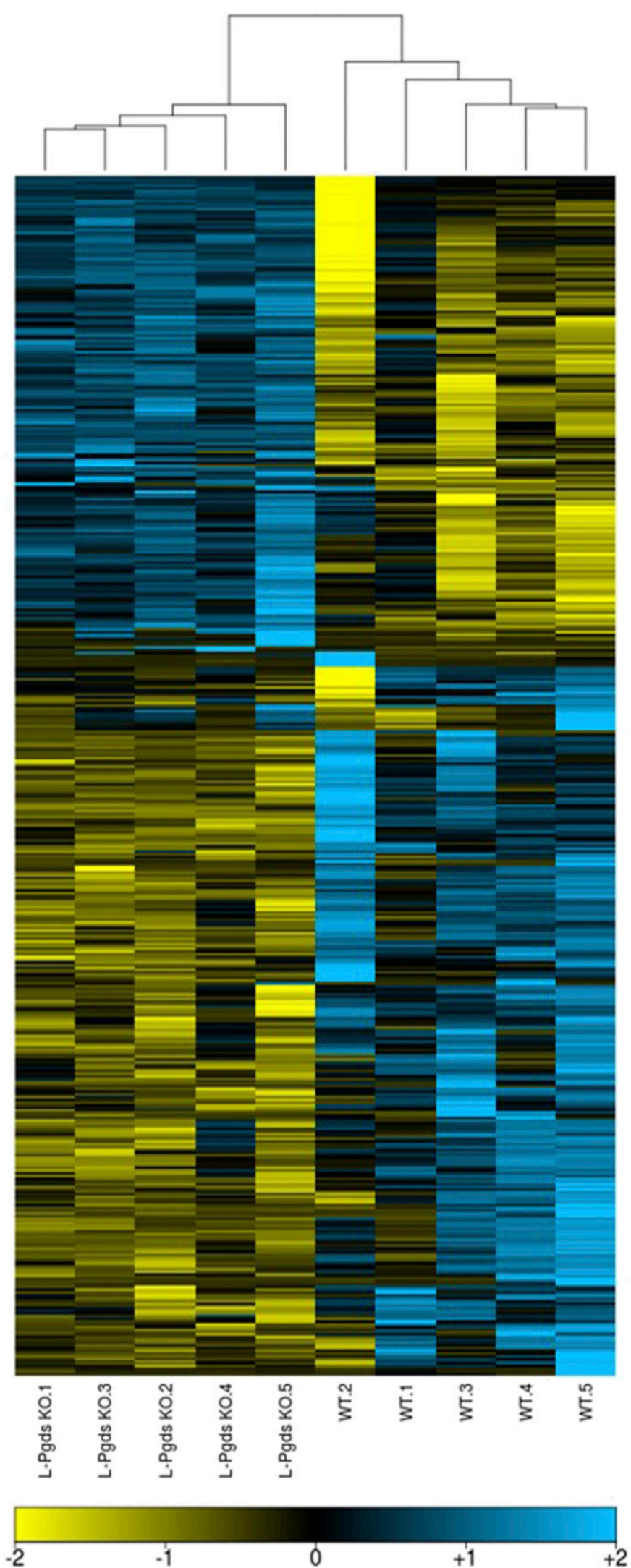

Fig. 5. Effect of L-Pgds deletion in mouse aorta. Heatmap representation of 391 genes differentially expressed $(q<0.1)$ between WT and L-Pgds KO male mice in aorta. female L-Pgds KO showed an increased systolic BP (Fig. 2, A and $\mathrm{B})$.

It has been reported that L-Pgds KO mice show features of the metabolic syndrome that correlates with hypothalamicpituitary-adrenal hyperactivity (Evans et al., 2013). Indeed, corticotropin-releasing hormone receptor $2(\mathrm{Crhr} 2)$ was upregulated 1.6-fold in the aorta of L-Pgds KO mice.

In addition, significant changes in the expression of a number of genes involved in glucose metabolism and insulin signaling were identified in the aorta of L-Pgds KO mice, including the upregulation of nicotamide nucleotide transhydrogenase (Nnt, fold change of 2.5) and the downregulation of insulin receptor (Insr, fold change of 1.5), insulin-degrading enzyme (Ide, fold change 1.4), lipin 1 (Lpin1, fold change of 1.6), Acyl-CoA synthetase medium-chain family member 3 (Acsm3, fold change of 1.6), acyl-CoA synthetase long-chain family member 4 (Acsl4, fold change of 1.8), and glycogen synthase-1 (Gys1, fold change of 1.3).

Changes in the expression of these genes are consistent with the insulin-resistant phenotype observed in the L-Pgds KO (Ragolia et al., 2005).

It is well established that insulin resistance contributes to endothelial dysfunction and a consequent predisposition to thrombosis in patients with diabetes. Increased oxidative stress may also contribute to this phenotype (Ceriello and Motz, 2004). Indeed, several aortic genes related to the oxidative stress response, such as heme oxygenase 1 (Hmox1, fold change of 1.4), glutathione S-transferase $\mu$ (Gstm2, fold change of 1.2), and thioredoxin-interacting protein (Txnip, fold change of 1.5) were dysregulated in L-Pgds KO mice.

Finally, Ingenuity Pathways Analysis revealed that the top canonical pathways enriched by genes differentially regulated between WT and L-Pgds KO mice are related to the cellular stress responses, such as "Protein ubiquitination pathway," "Unfolded protein response," "NRF2-mediated oxidative stress response," and "Production of nitric oxide and reactive oxygen species in macrophages" (as shown in Fig. 7). Interestingly, GO enrichment analysis revealed that all the top 10 molecular functions enriched by genes differentially regulated between WT and L-Pgds KO mice are related to protein-binding function (Fig. 8), and about 6\% of DEGs are transporters, and most of them are members of the solute carrier group of membrane transport proteins (solute carrier family 4, 7, 11, 12, 22, 25, 26, 39, 43, 50; Supplemental Table 1).

Gene expression profiles of L-PgdsS and H-Pgds are summarized in Supplemental Table 1.

\section{Discussion}

Both human and mouse studies indicate an important role of $\mathrm{PGD}_{2}$ in the vasculature.

In this study, we demonstrated that the systemic biosynthesis of $\mathrm{PGD}_{2}$ is generated mainly through H-Pgds (about $90 \%$ ) and only partially through L-Pgds (Fig. 1). However, deletion of L-Pgds, but not H-Pgds, elevated systemic BP (Fig. 2) and accelerated the thrombogenic occlusive response to a photochemical injury to the carotid artery in mice (Fig. 3). Thus, the effect of L-Pgds deletion on these cardiovascular phenotypes was not directly or quantitatively related to the suppression of systemic $\mathrm{PGD}_{2}$ biosynthesis. In fact, HQL-79, a H-PGDS inhibitor, further depresses urinary PGDM in L-Pgds KO mice, but has no incremental effect on BP level 

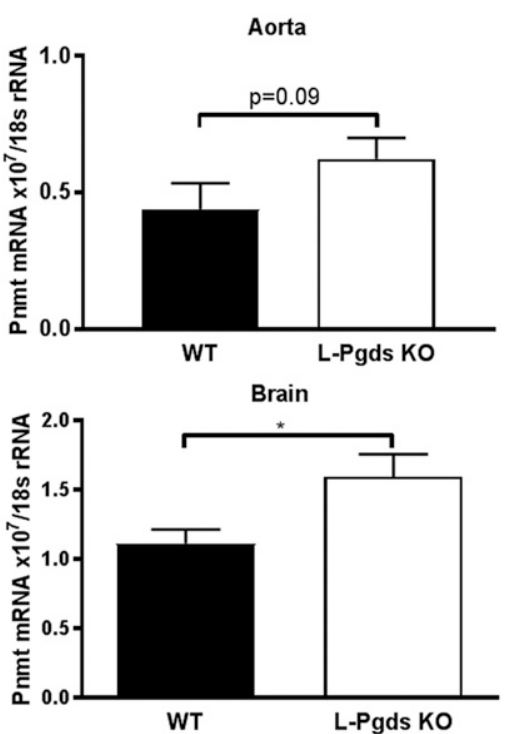

WT
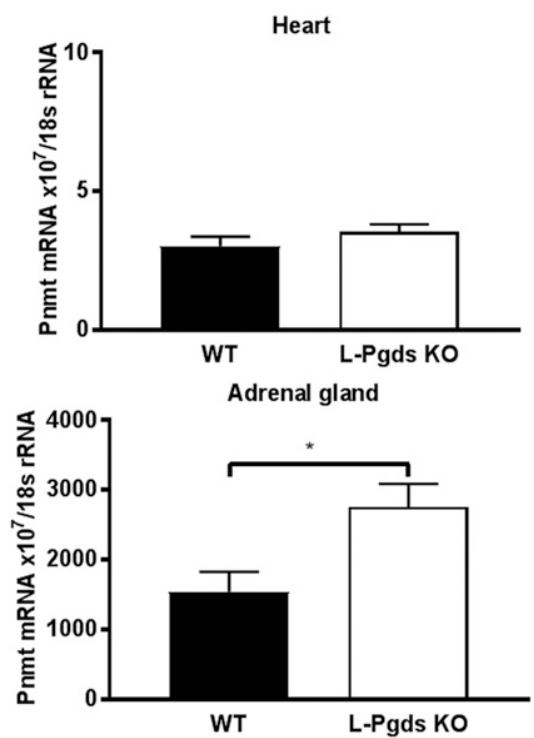

Fig. 6. Effect of L-Pods deletion on Pnmt expression. Expression of Pnmt in aorta, heart, brain, and adrenal gland in WT and L-Pgds male KO mice measured by reverse-transcription polymerase chain reaction. Data are mean \pm S.E.M., $n=5 ; * P<0.05$. or the thrombogenic response in mice (Fig. 4). Thus, although it is known that $\mathrm{PGD}_{2}$ can induce relaxation of vascular and nonvascular smooth muscle cells (Narumiya and Toda, 1985; Braun and Schror, 1992) and inhibit platelet activation and aggregation by increasing adenylate cyclase activity (Bushfield et al., 1985), we failed to observe any effect of $\mathrm{H}$-Pgds deletion, the major contributor of systemic production of $\mathrm{PGD}_{2}$, on $\mathrm{BP}$ or thrombosis.

Previously, L-PGDS had been linked to BP homeostasis: both serum and urinary levels of L-PGDS are much higher in patients with hypertension than those in normotensive subjects (Hirawa et al., 2002). Moreover, increased platelet $\mathrm{P}$-selectin expression, an index of platelet activation, is negatively correlated with a decrease in serum L-PGDS after percutaneous transluminal coronary angioplasty in the coronary sinus (Inoue et al., 2001).

L-PGDS, a member of the lipocalin superfamily, functions as a $\mathrm{PGD}_{2}$-producing enzyme and as a lipophilic carrier protein in the extracellular environment, binding several molecules, such as retinoids, thyroid hormones, bile pigments, fatty acids, hemoglobin, and cannabinoid metabolites (Herlong and Scott, 2006; Zhou et al., 2010; Orenes-Piñero et al., 2013, Elmes et al., 2018). Recently, it has also been reported that L-PGDS can bind hydrophilic ligands like NADPH (Qin et al., 2015).

The role of L-PGDS as lipophilic carrier protein has already been linked to the insulin-resistant and atherosclerotic phenotypes observed in L-Pgds KO mice (Ragolia et al., 2005).

The role of L-PGDS as a retinoid transporter might be involved in this process because a link exists between the retinoid X receptor and adipogenesis (Ragolia et al., 2005).

In this work, an unbiased analysis of the aortic transcriptomic profile revealed that genes relevant to $\mathrm{BP}$ and thrombosis are dysregulated in L-Pgds KO mice compared with the controls (Supplemental Table 1). Pnmt was one of the genes most highly overexpressed in the aorta (and other tissues relevant to catecholamine biosynthesis, such as brain and adrenal glands) of L-Pgds-deficient mice (Fig. 6).

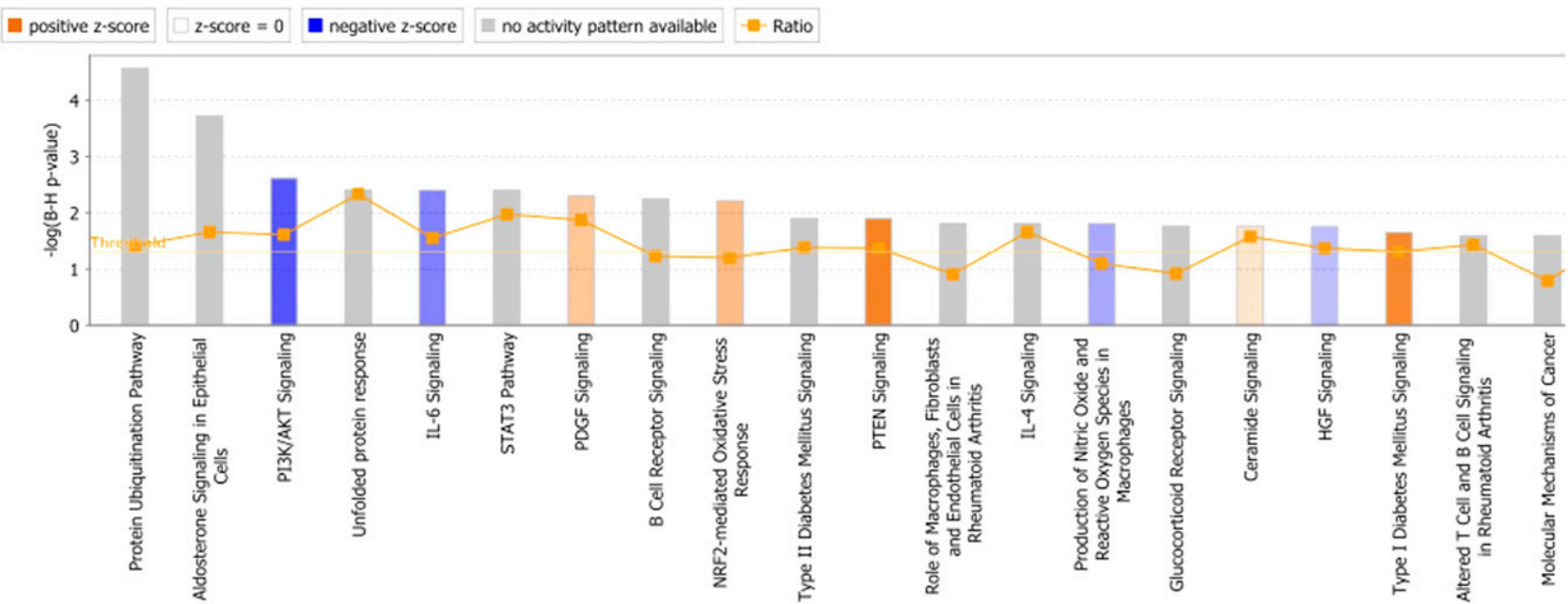

Fig. 7. Ingenuity Pathway Analysis. Top 20 canonical pathway enriched by genes differentially expressed $(q<0.1)$ between WT and L-Pgds KO mice in aorta. 


\begin{tabular}{|l|c|c|}
\hline GO molecular function complete & Fold Enrichment & P-value \\
\hline binding (GO:0005488) & 1.54 & $2.85 \mathrm{E}-31$ \\
\hline protein binding (GO:0005515) & 1.87 & $1.07 \mathrm{E}-29$ \\
\hline heterocyclic compound binding (GO:1901363) & 1.78 & $1.18 \mathrm{E}-11$ \\
\hline organic cyclic compound binding (GO:0097159) & 1.76 & $2.04 \mathrm{E}-11$ \\
\hline enzyme binding (GO:0019899) & 2.39 & $1.10 \mathrm{E}-08$ \\
\hline nucleoside phosphate binding (GO:1901265) & 2.18 & $4.23 \mathrm{E}-08$ \\
\hline nucleotide binding (GO:0000166) & 2.18 & $4.23 \mathrm{E}-08$ \\
\hline carbohydrate derivative binding (GO:0097367) & 2.2 & $4.60 \mathrm{E}-08$ \\
\hline small molecule binding (GO:0036094) & 2.04 & $1.70 \mathrm{E}-07$ \\
\hline receptor binding (GO:0005102) & 2.45 & $2.46 \mathrm{E}-07$ \\
\hline
\end{tabular}

Fig. 8. GO enrichment analysis. Top 10 GO molecular function enriched by genes differentially expressed $(q<0.1)$ between WT and L-Pgds KO male mice in aorta.
Previously, it has been reported that deletion of L-Pgds accelerates glucose intolerance and insulin resistance (Ragolia et al., 2005). Indeed, several genes involved in glucose metabolism and insulin signaling were differentially regulated in the aorta of L-Pgds KO mice compared with WT mice, including upregulation of Nnt and downregulation of Insr, Lipin 1, Acsm3, Acsl4, and Gys1. Moreover, Ingenuity Pathway Analysis revealed that pathways related to cellular stress responses such as "Protein ubiquitination pathway," "Unfolded protein response," "NRF2-mediated oxidative stress response," and "Production of nitric oxide and reactive oxygen species in macrophages" (as shown in Fig. 7) were enriched in those genes differentially expressed in WT and L-Pgds KO mice. These data are consistent with the hypothesis that insulin resistance in L-Pgds $\mathrm{KO}$ mice might result in oxidative stress in the vasculature, resulting in the hypertension and exaggerated thrombogenesis that we observed.

Although suppression of $\mathrm{PGD}_{2}$ biosynthesis by deletion of $\mathrm{H}-\mathrm{Pgds}$ has no resultant cardiovascular phenotype, this might be expected, given the absence of expression of its transcript in vascular cells. Although the phenotypes consequent to deletion of L-Pgds may reflect its actions independent of $\mathrm{PGD}_{2}$, it is possible that L-Pgds confers $\mathrm{PGD}_{2}$-dependent cardioprotection via an autocrine effect in the vasculature. Off-target effects, particularly mediated via its transporter function, may also confer cardioprotection, alone or in conjunction with an impact on $\mathrm{PGD}_{2}$. Indeed, $6 \%$ of the 391 unique genes differentially expressed in the aorta of L-Pgds-deficient mice compared with control mice are transporters, and most of them are members of the solute carrier group of membrane transport proteins (Supplemental Table 1). Moreover, the GO enrichment analysis revealed that all the top molecular functions enriched by genes differentially regulated between WT and L-Pgds KO mice are related to binding functions (Fig. 8).

In conclusion, these results indicate that the functional impact of L-Pgds deletion on vascular homeostasis may result from an autocrine effect of L-PGDS-dependent $\mathrm{PGD}_{2}$ on the vasculature and/or the L-PGDS function as lipophilic carrier protein.

\section{Acknowledgments}

We thank Nicholas Lahens, Helen Zou, Wenxuan Li, Weili Yan, and Faith Coldren for technical help and advice.

\section{Authorship Contributions}

Participated in research design: Song, Ricciotti, FitzGerald.

Conducted experiments: Song, Ricciotti, Liang.

Contributed new reagents or analytic tools: Song, Ricciotti.
Performed data analysis: Song, Ricciotti, Grosser, Grant.

Wrote or contributed to the writing of the manuscript: Song, Ricciotti, FitzGerald.

\section{References}

Aritake K, Kado Y, Inoue T, Miyano M, and Urade Y (2006) Structural and functional characterization of HQL-79, an orally selective inhibitor of human hematopoietic prostaglandin D synthase. J Biol Chem 281:15277-15286.

Braun M and Schrör K (1992) Prostaglandin D2 relaxes bovine coronary arteries by endothelium-dependent nitric oxide-mediated cGMP formation. Circ Res 71: $1305-1313$

Brunner HI, Bennett MR, Gulati G, Abulaban K, Klein-Gitelman MS, Ardoin SP, Tucker LB, Rouster-Stevens KA, Witte D, Ying J, et al. (2017) Urine biomarkers to predict response to lupus nephritis therapy in children and young adults. $J$ Rheumatol 44:1239-1248.

Bushfield M, McNicol A, and MacIntyre DE (1985) Inhibition of platelet-activatingfactor-induced human platelet activation by prostaglandin D2: differential sensitivity of platelet transduction processes and functional responses to inhibition by cyclic AMP. Biochem $J$ 232:267-271.

Ceriello A and Motz E (2004) Is oxidative stress the pathogenic mechanism underlying insulin resistance, diabetes, and cardiovascular disease? The common soil hypothesis revisited. Arterioscler Thromb Vasc Biol 24:816-823.

Cheng Y, Wang M, Yu Y, Lawson J, Funk CD, and Fitzgerald GA (2006) Cyclooxygenases, microsomal prostaglandin E synthase-1, and cardiovascular function. $J$ Clin Invest 116:1391-1399.

Duan B, Zhang L, Ding X, Li L, Li Y, Geng H, and Ma Y (2016) Serum beta-trace protein as a novel predictor of pregnancy-induced hypertension. J Clin Hypertens (Greenwich) 18:1022-1026.

Eguchi Y, Eguchi N, Oda H, Seiki K, Kijima Y, Matsu-ura Y, Urade Y, and Hayaishi O (1997) Expression of lipocalin-type prostaglandin D synthase ( $\beta$-trace) in human heart and its accumulation in the coronary circulation of angina patients. Proc Natl Acad Sci USA 94:14689-14694.

Elmes MW, Volpe AD, d'Oelsnitz S, Sweeney JM, and Kaczocha M (2018) Lipocalintype prostaglandin D synthase is a novel phytocannabinoid-binding protein. Lipids 53:353-360.

Evans JF, Islam S, Urade Y, Eguchi N, and Ragolia L (2013) The lipocalin-type prostaglandin D2 synthase knockout mouse model of insulin resistance and obesity demonstrates early hypothalamic-pituitary-adrenal axis hyperactivity. $J$ Endocrinol 216:169-180.

Goh Y, Nakajima M, Azuma I, and Hayaishi O (1988) Prostaglandin D2 reduces intraocular pressure. $\mathrm{Br} J$ Ophthalmol 72:461-464.

Grant GR, Liu J, and Stoeckert CJ Jr (2005) A practical false discovery rate approach to identifying patterns of differential expression in microarray data. Bioinformatics 21:2684-2690.

Hall IP (2000) Second messengers, ion channels and pharmacology of airway smooth muscle. Eur Respir J 15:1120-1127.

Herlong JL and Scott TR (2006) Positioning prostanoids of the D and J series in the immunopathogenic scheme. Immunol Lett 102:121-131.

Hirai H, Abe H, Tanaka K, Takatsu K, Sugamura K, Nakamura M, and Nagata K (2003) Gene structure and functional properties of mouse CRTH2, a prostaglandin D2 receptor. Biochem Biophys Res Commun 307:797-802.

Hirawa N, Uehara Y, Yamakado M, Toya Y, Gomi T, Ikeda T, Eguchi Y, Takagi M, Oda H, Seiki K, et al. (2002) Lipocalin-type prostaglandin D synthase in essential hypertension. Hypertension 39:449-454

Inoue T, Eguchi Y, Matsumoto T, Kijima Y, Kato Y, Ozaki Y, Waseda K, Oda H, Seiki $\mathrm{K}$, Node K, et al. (2008) Lipocalin-type prostaglandin D synthase is a powerful biomarker for severity of stable coronary artery disease. Atherosclerosis 201: 385-391.

Inoue T, Takayanagi K, Morooka S, Uehara Y, Oda H, Seiki K, Nakajima H, and Urade Y (2001) Serum prostaglandin D synthase level after coronary angioplasty may predict occurrence of restenosis. Thromb Haemost 85:165-170.

Kabashima K and Narumiya S (2003) The DP receptor, allergic inflammation and asthma. Prostaglandins Leukot Essent Fatty Acids 69:187-194.

Miyagi M, Miwa Y, Takahashi-Yanaga F, Morimoto S, and Sasaguri T (2005) Activator protein-1 mediates shear stress-induced prostaglandin D synthase gene expression in vascular endothelial cells. Arterioscler Thromb Vasc Biol 25:970-975.

Mohri I, Aritake K, Taniguchi H, Sato Y, Kamauchi S, Nagata N, Maruyama T, Taniike M, and Urade Y (2009) Inhibition of prostaglandin D synthase suppresses muscular necrosis. Am J Pathol 174:1735-1744. 
Nagoshi H, Uehara Y, Kanai F, Maeda S, Ogura T, Goto A, Toyo-oka T, Esumi H, Shimizu T, and Omata M (1998) Prostaglandin D2 inhibits inducible nitric oxide synthase expression in rat vascular smooth muscle cells. Circ Res 82:204-209.

Narumiya S and Toda N (1985) Different responsiveness of prostaglandin D2sensitive systems to prostaglandin D2 and its analogues. $\mathrm{Br}$ J Pharmacol 85: $367-375$

Negoro H, Soo Shin W, Hakamada-Taguchi R, Eguchi N, Urade Y, Goto A, Toyo-Oka T, Fujita T, Omata M, and Uehara Y (2002) Endogenous prostaglandin D2 synthesis reduces an increase in plasminogen activator inhibitor-1 following in terleukin stimulation in bovine endothelial cells. J Hypertens 20:1347-1354.

Orenes-Piñero E, Manzano-Fernández S, López-Cuenca Á, Marín F, Valdés M, and Januzzi JL (2013) $\beta$-Trace protein: from GFR marker to cardiovascular risk predictor. Clin J Am Soc Nephrol 8:873-881.

Qin S, Shimamoto S, Maruno T, Kobayashi Y, Kawahara K, Yoshida T, and Ohkubo T (2015) Thermodynamic and NMR analyses of NADPH binding to lipocalin-type prostaglandin D synthase. Biochem Biophys Res Commun 468:234-239.

Ragolia L, Palaia T, Hall CE, Maesaka JK, Eguchi N, and Urade Y (2005) Accelerated glucose intolerance, nephropathy, and atherosclerosis in prostaglandin D2 synthase knock-out mice. J Biol Chem 280:29946-29955.

Reja V, Goodchild AK, and Pilowsky PM (2002) Catecholamine-related gene expression correlates with blood pressures in SHR. Hypertension 40:342-347.

Ricciotti E and FitzGerald GA (2011) Prostaglandins and inflammation. Arterioscler Thromb Vasc Biol 31:986-1000.

Sawyer N, Cauchon E, Chateauneuf A, Cruz RP, Nicholson DW, Metters KM, O'Neill GP, and Gervais FG (2002) Molecular pharmacology of the human prostaglandin D2 receptor, CRTH2. Br J Pharmacol 137:1163-1172.

Song WL, Lawson JA, Wang M, Zou H, and FitzGerald GA (2007) Noninvasive assessment of the role of cyclooxygenases in cardiovascular health: a detailed HPLC/MS/MS method. Methods Enzymol 433:51-72.

Song WL, Stubbe J, Ricciotti E, Alamuddin N, Ibrahim S, Crichton I, Prempeh M, Lawson JA, Wilensky RL, Rasmussen LM, et al. (2012) Niacin and biosynthesis of $\mathrm{PGD}_{2}$ by platelet COX-1 in mice and humans. J Clin Invest 122: 1459-1468.

Taba Y, Sasaguri T, Miyagi M, Abumiya T, Miwa Y, Ikeda T, and Mitsumata M (2000) Fluid shear stress induces lipocalin-type prostaglandin $\mathrm{D}(2)$ synthase expression in vascular endothelial cells. Circ Res 86:967-973.

Tanaka R, Miwa Y, Mou K, Tomikawa M, Eguchi N, Urade Y, Takahashi-Yanaga F, Morimoto S, Wake N, and Sasaguri T (2009) Knockout of the l-pgds gene aggravates obesity and atherosclerosis in mice. Biochem Biophys Res Commun 378: $851-856$.

Urade Y and Eguchi N (2002) Lipocalin-type and hematopoietic prostaglandin D synthases as a novel example of functional convergence. Prostaglandins Other Lipid Mediat 68-69:375-382.

Urade Y and Hayaishi O (2000) Prostaglandin D synthase: structure and function. Vitam Horm 58:89-120.

White CA, Ghazan-Shahi S, and Adams MA (2015) $\beta$-Trace protein: a marker of GFR and other biological pathways. Am J Kidney Dis 65:131-146.

Yalcin MU, Gurses KM, Kocyigit D, Kesikli SA, Tokgozoglu L, Guc D, Aytemir K, and Ozer N (2016) Elevated serum beta-trace protein levels are associated with the presence of atrial fibrillation in hypertension patients. J Clin Hypertens (Greenwich) 18:439-443.

Zhou Y, Shaw N, Li Y, Zhao Y, Zhang R, and Liu ZJ (2010) Structure-function analysis of human l-prostaglandin D synthase bound with fatty acid molecules. FASEB J 24:4668-4677.

Address correspondence to: Dr. Garret A. FitzGerald, Department of Systems Pharmacology and Translational Therapeutics, Perelman School of Medicine, University of Pennsylvania, Translational Research Center, 3400 Civic Center Boulevard, Building 421, 10th Floor, Room 116, Philadelphia, PA 19104-5158. E-mail: garret@upenn.edu 